\title{
Pleural Cholesterol to the Diagnosis of Exudative Effusion
}

\author{
Rogério Rufino*, Bruna L. Marques, Renato de Lima Azambuja, \\ Thiago Mafort, José G. Pugliese and Cláudia Henrique da Costa
}

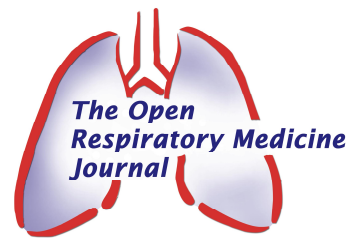

State University of Rio de Janeiro, Pulmonology Department, Rio de Janeiro, 20551-030, Brazil

\begin{abstract}
Introduction: Diagnostic approaches to patients with a pleural effusion must be precise because many procedures depend on the nature of the fluid in the effusion. To date, no biochemical test is considered an appropriate alternative to Light's criteria. This study compared the absolute pleural cholesterol (PC) level and the pleural cholesterol/serum cholesterol (PC/SC) ratio with Light's criteria to determine exudative pleural effusions.
\end{abstract}

Materials and Methods: This study was a case series of 100 consecutive patients with pleural effusions. The clinical parameters that were used to diagnosis an exudative effusion included the cholesterol level, a pleural cholesterol level $\geq$ $50 \mathrm{mg} / \mathrm{dL}$, a pleural/serum ratio $\geq 0.4$, and Light's criteria. The sensitivity, specificity, and positive and negative predictive values of each test for the diagnosis of an exudative effusion were assessed.

Results: A total of 79 patients were definitively diagnosed with an exudative effusion and were included in the trial and analyzed. The mean PC level in the exudates was $90.39 \mathrm{mg} / \mathrm{dL}$. The PC levels demonstrated a sensitivity of $97.22 \%$, a specificity of $85.71 \%$, a positive predictive value of $98.59 \%$ and a negative predictive value of $75 \%$. The $\mathrm{PC} / \mathrm{SC}$ ratio demonstrated a sensitivity of $81.48 \%$, a specificity of $57.14 \%$, a positive predictive value of $93.61 \%$ and a negative predictive value of $28.57 \%$.

Conclusions: The pleural cholesterol dosage level and the pleural/serum cholesterol ratio can be utilized as unique biomarkers to identify an exudative effusion and replace Light's criteria.

Keywords: Cholesterol, exudative effusion, pleural effusion.

\section{INTRODUCTION}

A pleural effusion (PE) is a frequent challenge in medical practice. A PE is an abnormal collection of fluid between the parietal and visceral pleurae. The following pathological mechanisms have been isolated or isolated in association, which are correlated with the development of a PE: a thoracic duct obstruction, a pleural permeability increase, a capillary pulmonary pressure increase, low oncotic pressure and low intrapleural pressure [1,2]. In such cases, pleural disease is present and is typically unilateral. A specific approach is needed to diagnose pleural disease, which is particularly important for infectious and neoplastic disease. Most PEs are a manifestation of a dysfunction or a distant clinical disease, such as hepatic, cardiac or renal failure. A $\mathrm{PE}$ diagnosis is established based on anamnesis and a physical exam, together with an imaging method that shows the pleural fluid collection $[1,2]$. Parameters, such as patient symptoms, clinical past diagnoses, and effusion volume, are essential in the medical decision to perform thoracentesis, which will categorize the effusion as a transudate or an exudate and help identify the etiology of the effusion [1-3].

Since 1972, a diagnosis of an exudative pleural effusion has been established when the fluid characteristics meet one of Light's criteria, which include a pleural/serum protein

*Address correspondence to this author at Avenida 28 de Setembro, 77 - Vila Isabel - Rio de Janeiro, Brazil; Fax: 55.21.2868-8248; E-mail: rrufino.uerj@gmail.com ratio $(\mathrm{PP} / \mathrm{SP})>0.5$, a pleural $/$ serum $\mathrm{LDH}$ level $>0.6$ and a pleural LDH level over $2 / 3$ of the reference value (or $200 \mathrm{IU}$ ) [4]. Few alternative biomarkers have been proposed to determine an exudative PE. Pleural cholesterol (PC) and the pleural/serum cholesterol ratio (PC/SC) have been found in the literature as alternative biomarkers to Light's criteria but without consistent data [5-7].

\section{OBJECTIVES}

General: To study PC and the PC/SC ratio as markers of an exudative pleural effusion.

Specific: To determine the specificity, sensitivity, and predictive values of pleural cholesterol in the diagnosis of exudative pleural effusions.

\section{MATERIALS AND METHODS}

This study was a case series of 100 consecutive patients with pleural effusions who attended the pleural disease center of a university hospital from March 2009 to March 2012.

\section{Inclusion Criteria}

1. Over 18 years of age.

2. Signed informed consent regarding the procedure.

3. Blood cell analysis and coagulation (TAP, PTT and INR), serum LDH, protein, glucose, amylase, bilirubin, cholesterol, and triglyceride tests. 
4. A chest radiograph with upright posteroanterior and lateral views and decubitus chest radiographs with a horizontal ray.

5. A diagnosis with the etiology of an exudative pleural effusion.

\section{Exclusion Criteria}

1. Hemodynamic instability.

2. Coagulopathies.

3. A bilateral pleural effusion with an established diagnosis of cardiac, hepatic or renal failure.

4. A unilateral, small-volume effusion, which would have complicated thoracentesis/pleural biopsy.

\section{Biochemical Markers Used to Diagnose an Exudative Pleural Effusion}

1. The effusion was considered to be exudative when the fluid properties met at least one of Light's criteria: a $\mathrm{PP} / \mathrm{SP}$ ratio $>0.5$; a pleural/serum $\mathrm{LDH}$ level $>0.6$ or a pleural LDH level over $2 / 3$ of the reference value (or $200 \mathrm{IU}$ ).

2. The effusion was considered to be exudative according to the following cholesterol criteria: $\mathrm{PC} \geq 50 \mathrm{mg} / \mathrm{dL}$ or a $\mathrm{PC} / \mathrm{SC}$ ratio $\geq 0.4$.

\section{Etiological Diagnosis}

In 62 cases, we performed pleural biopsy using a Cope's needle. The effusion was considered malignant when there was positive cytopathology or a positive histopathology analysis. A tuberculosis diagnosis was made when the adenosine deaminase (ADA) level was over $40 \mathrm{U} / \mathrm{L}$, acidfast bacilli were found, a positive Mycobacterium sp. culture was detected in the fluid or tissue, or caseous granulomas were present according to the histopathological analysis.

\section{Statistical Analysis}

Only exudative pleural effusions with a final diagnosis of the etiology (gold standard) were included in the statistical analysis. Undetermined pleural effusions were not included in the analysis. The specificity, sensitivity and positive and negative predictive values of $\mathrm{PC}$ and the $\mathrm{PC} / \mathrm{SC}$ ratio for the diagnosis of an exudative pleural effusion were determined. The agreement between Light's criteria and PC and the $\mathrm{PC} / \mathrm{SC}$ ratio was analyzed, and each of the parameters in Light's criteria were analyzed. A ROC curve was generated using GraphPad Prism 6.0.

\section{ETHICS}

This study adhered to the current laws of Brazil and ethical practice in research. All of the patients consented to the procedure, and this study was approved by the Ethics Committee of Pedro Ernesto University Hospital, Brazil, number 2612.

\section{RESULTS}

Of 100 patients who were screened, 79 met the inclusion criteria and were definitively diagnosed with an exudative pleural effusion when the effusion was analyzed based on Light's criteria. There were 34 females (43\%) and 45 males
$(57 \%)$. The median age was 54 years (range $=18-84$ years). Overall, 48 patients were diagnosed with tuberculosis $(61 \%)$, 25 patients with cancer $(32 \%), 2$ patients with empyema (3\%), 1 patient with chylothorax $(1 \%), 1$ patient with amyloidosis (1\%), 1 patient with a pancreatic pleural effusion (1\%) and 1 patient with non-tuberculosis mycobacteriosis (1\%) (Table 1). The mean pleural cholesterol level in the exudates was $90.39 \mathrm{mg} / \mathrm{dL}$. Overall, 18 patients had a serum cholesterol level $\geq 200 \mathrm{mg} / \mathrm{dL}$. When each parameter was individually compared to Light's criteria, the following percent errors occurred in the diagnosis of the exudates: $5.97 \%$ for the $\mathrm{LDH}$ ratio, $7.04 \%$ for the protein ratio, $2.7 \%$ for $\mathrm{PC}$, and $18.51 \%$ for the cholesterol ratio. $\mathrm{PC}$ had a sensitivity of $97.22 \%$, a specificity of $85.71 \%$, a positive predictive value of $98.59 \%$ and a negative predictive value of $75 \%$. The $\mathrm{PC} / \mathrm{SC}$ ratio indicated a sensitivity of $81.48 \%$, a specificity of $57.14 \%$, a positive predictive value of $93.61 \%$ and a negative predictive value of $28.57 \%$ (Table 2 ).

Table 1. Causes of Lung Effusion

\begin{tabular}{|c|c|c|}
\hline Causes of Exudate Effusion (n) & Absolute (n) & (\%) \\
\hline \hline Tuberculosis & 48 & 61 \\
\hline Neoplasia & 25 & 32 \\
\hline Empyema & 2 & 3 \\
\hline Mycobacterium avium & 1 & $<1$ \\
\hline Chylothorax & 1 & $<1$ \\
\hline Amyloidosis & 1 & $<1$ \\
\hline Pancreas pleural effusion & 1 & $<1$ \\
\hline Total & 79 & 100 \\
\hline
\end{tabular}

Table 2. Comparative Analysis for Exudate Criteria

\begin{tabular}{|c|c|c|c|c|}
\hline & Sensibility & Specificity & False Positive & False Negative \\
\hline \hline PC & $97.22 \%$ & $85.71 \%$ & $98.59 \%$ & $75 \%$ \\
\hline PC/SC & $81.48 \%$ & $57.14 \%$ & $93.61 \%$ & $28.57 \%$ \\
\hline
\end{tabular}

Legend: $\mathrm{PC}=$ pleural cholesterol; $\mathrm{SC}=$ serum cholesterol.

The cut-off for the diagnosis of the exudates using PC and the $\mathrm{PC} / \mathrm{SC}$ ratio was determined (Table 3 ). Using Light's criteria (the $\mathrm{LDH}$ ratio) as a reference and the $\mathrm{PC}$ values, an ROC curve was generated (Fig. 1). In an ROC curve, the true positive rate (sensitivity) is plotted as a function of the falsepositive rate (specificity) for different cut-off points. Each point on the ROC curve represents a sensitivity/specificity pair that corresponds to a particular decision threshold. A test with perfect discrimination (no overlap between the two distributions) has an ROC curve that passes through the upper left corner (100\% sensitivity and 100\% specificity). The bias using the Bland-Altman plot was as follows: for $\mathrm{PC} / \mathrm{SC}$ and $\mathrm{pLDH} / \mathrm{sLDH}, 1.93$ (SD 5.24), with limits of agreement (LA) from -8.36 to 12.19 ; for $\mathrm{PC} / \mathrm{SC}$ and $\mathrm{PP} / \mathrm{SP}$, 0.11 (SD 0.17), with LA from -0.23 to 0.46 ; for $\mathrm{PC}$ and pLDH, -1097 (SD 4138), with LA from -9207 to 7012 (Fig. 2). 
Table 3. Different Cut Off Values for Determining Exudative Effusion

\begin{tabular}{|c|c|c|c|c|c|}
\hline PC & Sensibility & Specificity & PC/SC & Sensibility & Specificity \\
\hline \hline 35 & $98.21 \%$ & $85.71 \%$ & 0.2 & $98.14 \%$ & $42.85 \%$ \\
\hline 40 & $98.21 \%$ & $85.71 \%$ & 0.25 & $96.29 \%$ & $42.85 \%$ \\
\hline 45 & $97.22 \%$ & $85.71 \%$ & 0.3 & $88.88 \%$ & $42.85 \%$ \\
\hline 50 & $97.22 \%$ & $85.71 \%$ & 0.35 & $88.88 \%$ & $57.14 \%$ \\
\hline 55 & $94.44 \%$ & $100 \%$ & 0.4 & $81.48 \%$ & $57.14 \%$ \\
\hline 60 & $93.05 \%$ & $100 \%$ & 0.45 & $77.77 \%$ & $57.14 \%$ \\
\hline 65 & $88.88 \%$ & $100 \%$ & 0.5 & $66.66 \%$ & $71.42 \%$ \\
\hline 70 & $87.50 \%$ & $100 \%$ & 0.55 & $59.25 \%$ & $85.71 \%$ \\
\hline
\end{tabular}

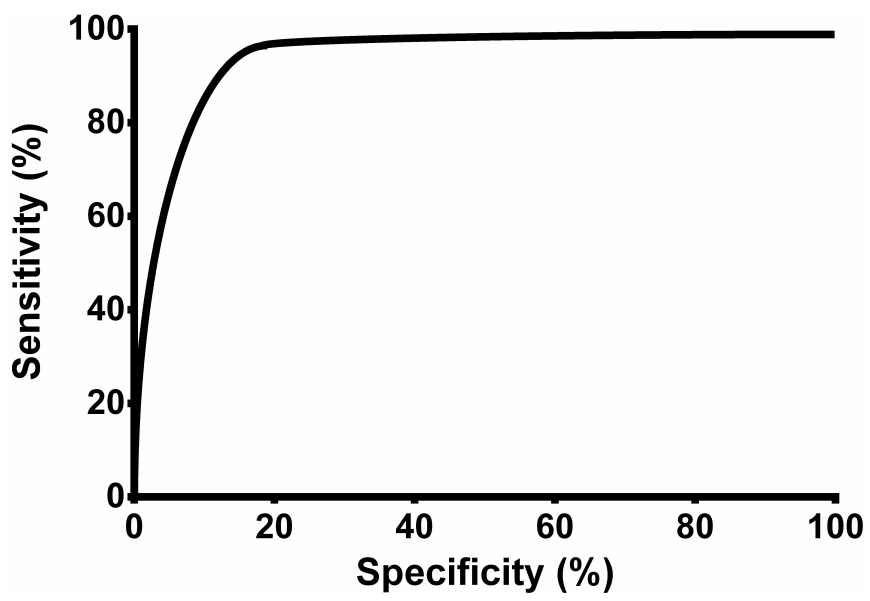

Fig. (1). Curve of the sensitivity and specificity of pleural cholesterol. In a Receiver Operating Characteristic (ROC) curve, the true positive rate (sensitivity) is plotted as a function of the false positive rate (specificity) for the different cut-off points. Each point on the ROC curve represents a sensitivity/specificity pair that corresponds to a particular decision threshold. A test with perfect discrimination (no overlap between the two distributions) has a ROC curve that passes through the upper left corner $(100 \%$ sensitivity and $100 \%$ specificity). Therefore, the closer the ROC curve is to the upper left corner, the higher the overall accuracy of the test. This ROC curve has an area of 0.871 (SD 0.027), a confidence interval of $95 \%(0.819-0.923)$, and $p<0.0001$.

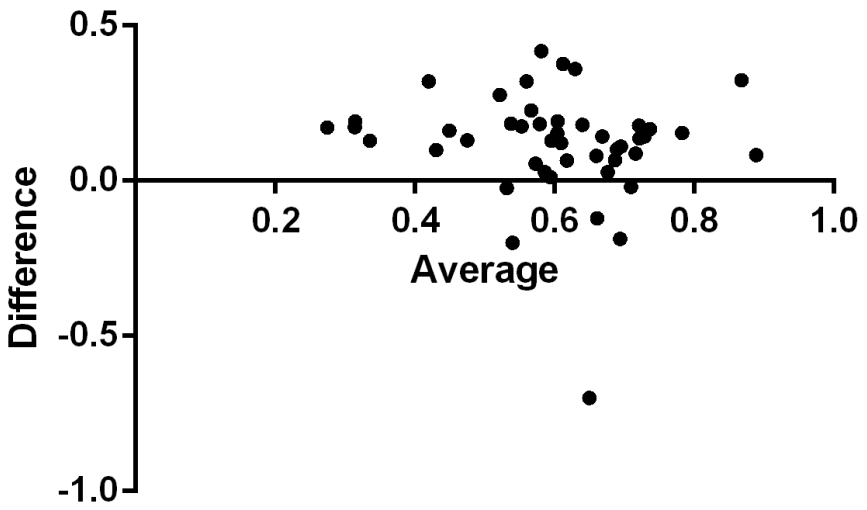

Fig. (2). Bland-Altman plot for $\mathrm{PC} / \mathrm{SC}$ and $\mathrm{PP} / \mathrm{SP}$. PC/SC could substitute $\mathrm{PP} / \mathrm{SP}$ in the determination of the exudates.

\section{DISCUSSION}

Since 1881, physicians have proposed that an etiological diagnosis of a pleural effusion can be made by thoracentesis and that the characterization of the effusion is essential to therapeutic decision making. In addition, the importance of the pleural protein level (1931) and the LDH level (1957) has been recognized. In 1972, Light et al. published a study in which exudative effusions could be distinguished from transudative effusions using both parameters with a high sensitivity and an acceptable specificity [4].

The sensitivity of Light's criteria is approximately $100 \%$ for exudates. However, up to $30 \%$ of transudates are misclassified as exudates according to these criteria. This misclassification has severe consequences for the patient and can lead to additional diagnostic approaches, such as subsequent thoracentesis with biopsy, surgery, or thoracoscopy [8-11]. False exudative effusions are associated with previous diuretic use, which alters serum and pleural biochemistry, including the cholesterol concentration. In addition, a high number of thoracentesis procedures leads to an increase in pleural LDH and contributes to a false exudative diagnosis.

Other parameters that have been studied as possible alternatives to Light's criteria include the serum-pleural albumin gradient and the NT-ProBNP [12].

A precise and fast analysis is necessary to treat patients with pleural effusions. Exudative effusions were correctly identified in this study. Light's criteria are superior to pleural cholesterol despite the high sensitivity and positive predictive value of both cholesterol parameters. The specificity of pleural cholesterol was lower than that in previous studies [13-16]. The use of a single pleural biochemical marker to diagnose a pleural effusion is important because additional tests may not be needed, which would improve the benefit/cost ratio. However, the pleural LDH and protein values can be used as prognostic markers in pleurodesis surgery for patients with a chronic symptomatic pleural effusion [2,8]. PC has not been evaluated for these patients. In this study, there were 13 patients with hypercholesterolemia. In these cases, the $\mathrm{PC} / \mathrm{SC}$ ratio can be used to offset the $\mathrm{PC}$ higher values.

\section{CONCLUSION}

The pleural cholesterol level can be used as an independent variable to diagnose an exudative effusion and can replace Light's criteria.

\section{CONFLICT OF INTEREST}

The authors did not have any conflict of interest to perform this article.

\section{ACKNOWLEDGEMENTS}

Declared none.

\section{REFERENCES}

[1] Sahn SA. The pleura: state of the art. Am Rev Resp Dis 1988; 138: 184-234.

[2] Light RW. Pleural effusions. Med Clin North 2011; 95: 1055-70.

[3] Light RW. Clinical practice: pleural effusion. N Engl J Med 2002; 346: 1971-7. 
[4] Light RW, MacGregor MI, Luchsinger PC, et al. Pleural effusions: the diagnostic separation of transudates and exudates. Ann Intern Med 1972; 77: 507-13.

[5] Guleria R, Argarwal SR, Sinha S, et al. Role of pleural fluid cholesterol in differentiating transudative from exsudative pleural effusion. Nat Med India 2003; 16: 64-9.

[6] Ortega L, Heredia JL, Armengol R, et al. The differential diagnosis between pleural exudates and transudates: the value of cholesterol. Med Clin 1991; 96: 367-70.

[7] Costa M, Quiroga T, Cruz E. Measurement of pleura fluid cholesterol and lactate dehydrogenase: a simple and accurate test of indicators for separating exudates from transudates. Chest 1995; 108: 1260-3.

[8] Hooper C, Lee YC, Maskell N, BTS Pleural, Guideline Group. Investigation of a unilateral pleural effusion in adults: British Thoracic Society Pleural Disease Guideline 2010. Thorax 2010; 65: ii4-17.

[9] Gásquez I, Porcel JM, Vives M. Comparative analyses of Light's criteria and other biochemical parameters for distinguishing transudates from exudates. Respir Med 1998; 92: 762-5.
[10] Romero-Candeira S, Fernández C, Martin C, Sanchez-Paya J, Hernandez L. Influence of diuretics on the concentration of proteins and other components of pleural transudates in patients with heart failure. Am J Med 2001; 110: 681-6.

[11] Romero- Candeira S, Hernandes L. The separation of transudate and transudate with particular reference to the protein gradient. Curr Opin Pulm Med 2004; 10: 294-8.

[12] Yorgancioglu A, Alpaydin AO, Yaman N, et al. Serum and pleural fluid N-Terminal-Pro-B-Type natriuretic peptide concentrations in the differential diagnosis of pleural effusions. Tüberküloz ve Toraks Dergisi 2011; 59: 1-7.

[13] Heffner JE, Highland K, Brown LK. A meta-analysis derivation of continuous likehood ratios for diagnosis pleural fluid exudates. Am J Respir Crit Care Med 2003; 167: 1591-9.

[14] Abramowitz Y, Simanovsky N, Goldstein MS, et al. Pleural effusion: characterization with $\mathrm{CT}$ attenuation values and $\mathrm{CT}$ appearance. AJR 2009; 192: 618-23.

[15] Porcel G, Light RW. Diagnostic approach to pleural effusion in adults. Am Fam Physician 2006; 73: 1211-20.

[16] Huggins JT. Chylothorax and cholesterol pleural effusion. Semin Respir Crit Care Med 2010; 31: 743-50. 